A numerical method for the computation of

Faber polynomials for starlike domains

N. Papamichael, Maria Joana Soares

and

N.S. Stylianopoulos 



\title{
A Numerical Method for the Computation of Faber Polynomials for Starlike Domains
}

N. Papamichael *, Maria Joana Soares $†$ and N.S. Stylianopoulos *

\begin{abstract}
We describe a simple numerical process (based on the Theodorsen method for conformal mapping ) for computing approximations to Faber polynomials for starlike domains.
\end{abstract}

Keywords : Faber polynomials, conformal mapping, starlike domains, Theodorsen method.

* Department of Mathematics and Statistics, Brunel University, Uxbridge, Middlesex UB8 3PH, U.K.

† Departamento de Matematica, Universidade do Minho, 4700 Braga, Portuga 
BRUNEL UNIVERSITY SEP 1991

LIBRARY 


\section{Introduction}

Faber polynomials have well-known classical applications as basis sets for polynomial and ational approximations in the complex plane. In addition, their study has received considerable atten-tion recently, in connection with the construction of efficient iterative methods for solving systems of linear algebraic equations (see eg. [4]).Thus, the problem of developing numerical methods for com-puting approximations to the Faber polynomials for a region in the complex plane is of considerable current interest. This paper is concerned with the study of one such numerical method.

Let $\Gamma$ be a Jordan curve in the complex $z$-plane, and let $\psi$ denote the conformal map of

$D_{E}:=\{\mathrm{w}:|\mathrm{w}|>1\}$ onto $\Omega_{E}:=$ Ext $\Gamma$ normalized by the conditions

$$
\psi(\infty)=\infty \text { and } \quad \psi^{\prime}(\infty)>0 .
$$

Also, let $\Phi$ denote the inverse conformal map $\Phi:=\Psi^{[-1]}$, from $\Omega_{E}$ onto $D_{E}$, and observe that the Laurent series expansions of $\psi$ and $\Phi$ at infinity are of the form

$$
\psi(\mathrm{w})=\mathrm{cw}+\mathrm{c}_{0}+\mathrm{c}_{1} / \mathrm{w}+\mathrm{c}_{2} / \mathrm{w}^{2}+\ldots,
$$

and

$$
\Phi(\mathrm{z})=\mathrm{bz}+\mathrm{b}_{0}+\mathrm{b}_{1} / \mathrm{z}+\mathrm{b}_{2} / \mathrm{z}^{2}+\ldots . \text { with } b=1 / \mathrm{c},
$$

where $c=\psi^{1}(\infty)$ is the capacity of the curve $\Gamma$, ie. c:=cap $\Gamma$. Then, the nth Faber polynomial $\mathrm{p}_{\mathrm{n}}(n=0,1,2, \ldots)$ for the domain $\Omega_{1}:=$ Int $\Gamma$ is the polynomial part of the Laurent series expansion at infinity of the function $\Phi^{\mathrm{n}}$.

Full details of the theory of Faber polynomials and their approximating properties can be found in the two books by Gaier [9, Chap.1, §6 ] and Henrici [12, §18.1-18.2 ] and the other important references cited there. Of particular interest to us here is the relation

$$
p_{n+1}(z)=\frac{1}{c}\left\{z p_{n}(z)-\sum_{k=0}^{n} c_{k} p_{n-k}(z)-n c_{n}\right\}, \quad n=0,1, \ldots .
$$

which can be used to determine recursively the Faber polynomials $p_{n}$. However, because of the pres-ence of the generally unknown mapping coefficients $c$ and $\mathrm{c}_{\mathrm{i}}$, the use of (1.3) has received very little attention from the computational point of view (see e.g. the remark made by Gaier in $[9, \mathrm{p} .44])$.

As far as we are aware the most recent references on the computation of Faber polynomials are the papers by Coleman and Smith [2] and Ellacott [5]. Of these, [2] concerns the computation of Faber polynomials in circular sectors, and involves the derivation of an explicit formula for the corresponding mapping function $\psi$. The paper also contains a recurrence formula for determining the mapping coefficients $c_{\mathrm{j}}$, but Coleman and Smith do not make use of (1.3) in their computations. They choose, instead, to compute the coefficients of the Faber polynomials directly from a standard integral representation that makes use of their formula for $\psi$. ( We note, however, that their recurrence formula was subsequently used by Ellacott and Saff [6], for computing Faber polynomials in circular sectors, by means of (1.3).) In [5], Ellacott suggests computing the coefficients of Faber polynomials, from their integral representation in terms of the mapping function $\Phi$, by using the fast Fourier 
transform (FFT). He also considers the recursive computation of the polynomials by means of (1.3), but only for cases where the boundary curve $\Gamma$ is a polygon. For this, he proposes using the Schwartz-Christoffel formula for determining the expansion of $\psi^{1}$ and hence, by termwise integration, that of $\psi$.

Regarding numerical experiments, all the examples of Faber polynomials that we have come across in the literature, including those of [2] and [5], are for regions for which the mapping function $\Phi$, or its inverse $\psi$, is known exactly. The purpose of the present paper is to consider a fully numeri cal technique, based on using the well-known Theodorsen integral equation method for computing approximations to the coefficients $c$ and $\mathrm{c}_{\mathrm{j}}$ of the mapping function $\psi$, and then determining recursively the corresponding approximate Faber polynomials by means of (1.3). Our main objective is to show, by means of numerical examples, that this technique is very well-suited for computing accurate approximations to the Faber polynomials corresponding to a wide class of starlike curves $\Gamma$.

\section{The numerical method}

Let $\alpha_{j}^{(n)}$ denote the coefficients of the Faber polynomial $p_{n}$, ie.

$$
\mathrm{p}_{\mathrm{n}}(\mathrm{z})=\sum_{\mathrm{j}=0}^{\mathrm{n}} \alpha_{\mathrm{j}}^{(\mathrm{n})} \mathrm{z}^{\mathrm{j}}
$$

Then, by substituting (2.1) in the recurrence relation (1.3) and comparing coefficients of like powers of $\mathrm{z}$, we obtain the following relations for $\mathrm{n} \geq 1$ :

$$
\begin{array}{r}
\alpha_{0}^{(n+1)}=-\frac{1}{c}\left\{\sum_{k=0}^{n-1} c_{k} \alpha_{0}^{(n-k)}+(n+1) c_{n}\right\}, \\
\alpha_{j}^{(n+1)}=\frac{1}{c}\left\{\alpha_{j-1}^{(n)}-\sum_{k=0}^{n-j} c_{k} \alpha_{j}^{(n-k)}\right\}, \quad j=1,2, \ldots, n, \\
\alpha_{n+1}^{(n+1)}=\frac{1}{c} \alpha_{n}^{(n)} .
\end{array}
$$

Since

$$
\alpha_{0}{ }^{(1)}=-\mathrm{c}_{0} / \mathrm{c} \text { and } \alpha_{1}^{(1)}=1 / \mathrm{c}
$$

the relations (2.2)-(2.5) can be used to determine recursively the coefficients of the Faber polynomials $p_{n}, n=2,3, \ldots$, in terms of the Laurent coefficients $c$ and $c_{j}, j=1,2, \ldots$, of the mapping function $\psi$.

Consider now the " auxiliary " function

$$
\Psi(\mathrm{w}):=\log \frac{\psi(\mathrm{w})}{\mathrm{w}},
$$

and observe that it has a Laurent expansion of the form

$$
\Psi(\mathrm{w})=\gamma_{0}+\gamma_{1} / \mathrm{w}+\gamma_{2} / \mathrm{w}^{2}+\ldots,
$$

where $\gamma_{0}=\log c$. Hence

$$
\psi^{1}(\mathrm{w})-\frac{1}{\mathrm{w}} \psi(\mathrm{w})=\psi(\mathrm{w})\left\{-\gamma_{1} / \mathrm{w}^{2}-2 \gamma_{2} / \mathrm{w}^{3}-\ldots\right\},
$$


which, by substituting the expansions of $\psi$ and $\psi^{1}$ and comparing coefficients of like powers of $w$, yields the following relations:

$$
\begin{gathered}
\mathrm{c}_{0}=\mathrm{c} \gamma_{1}, \\
\mathrm{c}_{\mathrm{n}}=\mathrm{c} \gamma_{\mathrm{n}+1}+\frac{1}{\mathrm{n}+1} \sum_{j=0}^{\mathrm{n}-1}(\mathrm{n}-\mathrm{j}) \mathrm{c}_{\mathrm{j}} \gamma_{\mathrm{n}-\mathrm{j}}, \quad \mathrm{n} \geq 1
\end{gathered}
$$

Since

$$
\mathrm{c}=\exp \gamma_{0}
$$

the relations (2.8)-(2.10) can be used to determine recursively the mapping coefficients $c$ and $c_{j}$ from the coefficients $\gamma_{j}$ in the expansion (2.7) of the auxiliary function $\Psi$.

The numerical method considered in this section is based on the use of the recurrence relations (2.2)-(2.5) and (2.8)-(2.10), and is motivated by the following observation concerning the computation of the coefficients $\gamma_{j}$ by means of the Theodorsen method.

Assume that the boundary curve $\boldsymbol{\Gamma}$ is starlike with respect to the origin and is given in polar coordinates by

$$
\Gamma:=\left\{z: z=\rho(\theta) \mathrm{e}^{\mathrm{i} \theta}, 0 \leq \theta \leq 2 \pi\right\},
$$

where $\rho$ is piecewise differentiable in $[0,2 \pi]$. Also let $\Theta$ denote the boundary correspondence function associated with the conformal map $\psi$, ie.

$$
\psi\left(\mathrm{e}^{\mathrm{i} \tau}\right)=\rho(\Theta(\tau)) \mathrm{e}^{\mathrm{i} \Theta(\tau)}
$$

Then, $\Theta$ satisfies the Theodorsen integral equation

$$
\Theta(\tau)=\tau-\mathrm{k}[\log \rho(\Theta)(\tau))]
$$

where $\mathrm{K}$ denotes the well-known operator for conjugation on the unit circle ( see e.g. Gaier [8, Kap. II] and Henrici [12, §16.8]).

In the basic Theodorsen method equation (2.12) is solved iteratively for $\Theta$, by using a Jacobi type iteration of the form

$$
\begin{gathered}
\Theta^{0}(\tau \tau=\tau, \\
\Theta^{\mathrm{k}+1}\left(\tau \tau=\tau-\mathrm{k}\left[\log \rho \mathrm{lo}^{\mathrm{k}}(\tau \tau)\right), \quad \mathrm{k}=0,1, \ldots,\right.
\end{gathered}
$$

In practice, this iteration is performed in discretized form where, at the $\mathrm{k}$ th step, the function $\log \rho\left(\Theta^{\mathrm{k}}(\mathrm{T})\right)$ is replaced by its trigonometric interpolating polynomial

$$
\mathrm{T}_{\mathrm{N}}\left(\tau \tau:=\frac{1}{2} \mathrm{a}_{0}^{(\mathrm{k})}+\sum_{\mathrm{j}=1}^{\mathrm{N}-1}\left(\mathrm{a}_{\mathrm{j}}{ }^{(\mathrm{k})} \operatorname{cosj} \tau+\mathrm{b}_{\mathrm{j}}{ }^{(\mathrm{k})} \operatorname{sinj} \tau\right)+\frac{1}{2} \mathrm{a}_{\mathrm{N}}{ }^{(\mathrm{k})} \cos \mathrm{N} \tau\right.
$$

of degree $N$ and nodes

$$
\tau_{r}:=r \pi / N, \quad r=0,1, \ldots, 2 N-1 .
$$

Then, because of the properties of the operator $\mathrm{K}$, the $(\mathrm{k}+\mathrm{l})$ th approximation to the boundary 
correspondence function is given, from (2.13), by

$$
\Theta_{N}^{(k+1)}(\tau)=\tau+\sum_{j=1}^{N-1}\left(b_{j}{ }^{(k)} \cos j \tau-a_{j}{ }^{(k)} \sin j \tau\right)-\frac{1}{2} a_{N}{ }^{(k)} \sin N \tau .
$$

The resulting discrete iteration can be stated as follows:

- Set $\Theta^{0}(\tau)=\tau$.

- Do steps (i) and (ii) with $k=0,1,2, \ldots$, until convergence:

(i) Compute the coefficients $a_{j}{ }^{(k)}$ and $b_{j}{ }^{(k)}$ of the trigonometric polynomial (2.14).

(ii) Use (2.16) to compute the values $\Theta_{N}^{(k+1)}\left(\tau_{r}\right), \quad r=0,1, \ldots 2 N-1$.

The coefficients $\mathrm{a}_{\mathrm{j}}{ }^{\mathrm{k})}, \mathrm{b}_{\mathrm{j}}{ }^{\mathrm{k})}$ in step (i) of the iteration can be computed efficiently in $O(N \log N)$ opera- ions by the use of the FFT. Similarly, in step (ii), the computations of the values $\Theta_{N}^{(k+1)}\left(\tau_{r}\right)$ can be performed by the use of the FFT. That is, the basic Theodorsen method involves the application of two FFTs in each iterative step.

As the above remarks indicate, the Theodorsen method is based essentially on approximating the coefficients of the trigonometric polynomial

$$
T_{N}(\tau)=\frac{1}{2} a_{0}+\sum_{j=1}^{N-1}\left(a_{j} \cos j \tau+b_{j} \sin j \tau\right)+\frac{1}{2} a_{N} \cos N \tau,
$$

which interpolates, the function $\log \rho \Theta(\tau)$ at the nodes (2.15). The relevance of this observation with our work here is that once the approximations $\bar{a}_{j}$ and $\bar{b}_{j}$ to $\mathrm{a}_{\mathrm{j}}$ and $\mathrm{b}_{\mathrm{j}}$ are found, then the corresponding approximations to the Laurent coefficients $\gamma_{j}, j=0,1, \ldots N$, of the auxiliary function $\Psi$ are given quite simply by

$$
\bar{\gamma}_{0}=\frac{1}{2} \bar{a}_{0} \quad \text { and } \quad \bar{\gamma}_{j}=\bar{a}_{j}+i \bar{b}_{j}, \quad j=1,2, \ldots, N
$$

with $b_{N}=0$ ( see e.g. [8,p.87] and [12, p.407] ). Thus, the details of the proposed algorithm are as follows :

STEP 1 : Use the Theodorsen method to compute approximations $\bar{a}_{j}, \bar{b}_{j}$ to the coefficients $a_{j}$, $b_{j}$. of the trigonometric polynomial (2.17). Hence, use (2.18) to compute the approximations $\bar{\gamma}_{j}, j=0,1, \ldots N$, to the Laurent coefficients of the auxiliary function $\boldsymbol{\Psi}$.

STEP 2 : Use the relations (2.8)-(2.10) to compute recursively the approximations to the mapping coefficients $c$ and $c_{j}, j=0,1, \ldots N$.

STEP 3 : Use the relations (2.2)-(2.5) to compute recursively approximations to the coefficients $\alpha_{j}^{(n)}$ of the Faber polynomials $p_{n}, n=I, 2, \ldots N$. 
We end this section by making the following remarks concerning the convergence of the Theodorsen method and our implementation of Step 1 of the algorithm outlined above.

It is well-known that convergence of the iteration (2.13), and of the corresponding discrete iteration defined by (2.16), can be guaranteed only if the curve (2.11) satisfies a so-called "Econdition ", which requires that

$$
\varepsilon:=\sup _{0 \leq \tau \leq 2 \pi}\left|\rho^{1}(\tau) / \rho(\tau)\right|<1 .
$$

It is also well-known that if (2.19) is violated, then convergence can often be recovered by using underrelaxation with appropriate relaxation factor. This approach of using the Jacobi iteration (2.16), with underrelaxation if necessary, is sufficient for the purpose of the present paper, ie. for illustrating the application of the algorithm outlined above. We note, however, that it might be more efficient to perform Step 1 of the algorithm by solving the discrete Theodorsen integral equation using one of the several alternative iterative procedures which have been proposed in recent years. Full details of these recent developments can be found in the survey paper by Gutknecht [11] and the other references cited there.

\section{Numerical examples}

In this section we present five examples illustrating the use of the numerical algorithm of Section 2, for computing approximations to Faber polynomials associated with starlike curves. For comparison purposes, in each of our examples we choose a curve $\boldsymbol{\Gamma}$ for which the mapping function $\psi$ is known exactly. Then, by using if necessary the symbolic manipulation package MAPLE, we determine in rational form the mapping coefficients $c$, $c_{j}$ and ( whenever $\phi:=\psi^{[-1]}$ is also available ) the corresponding exact Faber polynomials.

All our numerical results were computed on a Pyramid 9825 computer, using programs written in double precision Fortran. ( Double length working on the Pyramid is between 15 and 16 significant figures. ) In presenting these results we give in each case the following information, regarding the use of the Theodorsen method in Step 1 of the algorithm:

- The degree $\mathrm{N}$ of the trigonometric interpolating polynomial used.

- The relaxation parameter $\omega$ used in the iteration.

- The number $K$ of iterations needed for convergence.

3.1 Ellipse Let $\mathrm{z}=x+i y$ and let $\Gamma$ be the ellipse

$$
\frac{x^{2}}{a^{2}}+\frac{y^{2}}{b^{2}}=1, \quad a>b>0 .
$$

Then,

$$
\psi(w)=\frac{1}{2}\left\{(a+b) w+(a-b) w^{-1}\right\}
$$

and hence 


$$
\phi(z)=\frac{1}{a+b}\left\{z+z\left(1-\frac{a^{2}-b^{2}}{z^{2}}\right)^{1 / 2}\right\} .
$$

In particular, if

$$
a=1+\mathrm{d} \quad \text { and } \quad b=1-\mathrm{d}, \quad 0<d<1
$$

, then

$$
\psi(w)=w+d / w
$$

and

$$
\phi(z)=\frac{1}{2}\left\{z+z\left(1-\frac{4 d}{z^{2}}\right)^{1 / 2}\right\}
$$

see e.g. [3, p.225] and [12, p.412]). Thus, in this case,

0 ,

$$
\mathrm{c}=1, \quad \mathrm{c}_{0}=0, \quad \mathrm{c}_{1}=d, \quad \mathrm{c}_{2}=\mathrm{c}_{3}=\ldots=
$$

and, for $n \geq 2$, the recurrence formula (1.3) simplifies to

$$
p_{n+1}(z)=z p_{n}(z)-d p_{n-1}(z)
$$

Hence

$$
\begin{gathered}
P_{1}(z)=z, \quad p_{2}(z)=z^{2}-2 d, \quad p_{3}(z)=z^{3}-3 d z, \\
P_{4}(z)=z^{4}-4 d z^{2}+2 d^{2}, \quad p_{5}(z)=z^{5}-5 d z^{3}+5 d^{2} z,
\end{gathered}
$$

e.t.c. More generally, it follows easily from the solution of the difference equation

that

$$
p_{n+1}-z p_{n}+d p_{n-1}=0
$$

$$
p_{j}(z)=\frac{1}{2^{j-1}} \sum_{k=0}^{[j / 2]}\left[\begin{array}{l}
j \\
2 k
\end{array}\right] z^{j-2 k}\left(z^{j-2 k}\left(z^{2}-4 d\right)^{k}, \quad j \geq 1,\right.
$$

where $[J]$ denotes the largest integer $\leq \mathrm{J}$ ( See $[3, \mathrm{p} .226]$ and note that due to a printing error the factor $2^{-\mathrm{j}+1}$ in the left hand side of (3.1) is given incorrectly in [3] as 2.)

In the case $d=0.4$, the Theodorsen method with $N=32$ and $\omega=0.5$ gives the following results, after $K=54$ iterations:

- The approximations to $c=1$ and $c_{1}=0.4$ are respectively

$$
1.0000000000024 \text { and } 0.4000000000010 \text {. }
$$

- The maximum error in the approximations to the coefficients

$$
{ }^{C} 0={ }^{C} 2={ }^{C} 3=--\cdot={ }^{C} 64=0
$$

is less than $7.9 \times 10^{-11}$. 
- The corresponding approximations to the nonzero coefficients

$$
\alpha_{0}{ }^{(10)}=-0.02048, \quad \alpha_{2}{ }^{(10)}=0.64, \quad \alpha_{4}{ }^{(10)}=-3.2,
$$

and

$$
\alpha_{6}^{(10)}=56, \quad \alpha_{8}^{(10)}=-4, \quad \alpha_{10}^{(10)}=1,
$$

of the Faber polynomial $p_{10}$ are respectively

$$
-0.02047999997, \quad-0.63999999998,-3.20000000012 \text {, }
$$

and

$$
5.60000000001,-3.99999999992,0.99999999998 \text {. }
$$

- The maximum error in the approximations to the coefficients

$$
\alpha_{1}{ }^{(10)}=\alpha_{3}{ }^{(10)}=\alpha_{5}{ }^{(10)}=\alpha_{7}^{(10)}=\alpha_{9}{ }^{(10)}=0,
$$

is less than $2.9 \times 10^{-15}$.

3.2 Two intersecting circles Let $\Omega_{1}$ be the domain bounded by the two intersecting circles

$$
|z-1.6|=2.0 \text { and }|z-0.9|=1.5 \text {. }
$$

That is $\Omega_{1}$, is bounded by the curve

$$
\Gamma:=\left\{z: z=\rho(\theta) \mathrm{e}^{\mathrm{i} \theta}, 0 \leq \theta \leq 2 \pi\right\},
$$

where

$$
\rho(\theta)=\left\{\begin{array}{rc}
0.9 \cos \theta+\left\{2.25-0.81 \sin ^{2} \theta\right\}^{1 / 2}, & 0 \leq \theta \leq \pi / 2, \\
-1.6 \cos \theta+\left\{4.00-2.56 \sin ^{2} \theta\right\}^{1 / 2}, & \pi / 2<\theta \leq \pi
\end{array}\right.
$$

With

$$
\rho(\theta)=\rho(2 \pi-\theta), \pi<\theta \leq 2 \pi
$$

Then

$$
\phi(z)=\frac{2}{5 z}\left(z-\frac{9}{10}\right)\left(z+\frac{8}{5}\right)
$$

and hence

$$
\psi(w)=\frac{5}{4} w-\frac{7}{20}+\frac{5}{4} w\left\{1-\frac{14}{25} \frac{1}{w}+\frac{1}{w^{2}}\right\}^{1 / 2},
$$

(see e.g. [13, p.48]). Thus, from (3.3),

$$
c=\frac{5}{2}, c_{o}=-\frac{7}{10}, c_{1}=\frac{72}{125}, \ldots . . . e . t . c .,
$$

and, from (3.2), the first four Faber polynomials are : 


$$
\begin{gathered}
p_{1}(z)=\frac{2}{5} z+\frac{7}{25}, \quad p_{2}(z)=\frac{4}{25} z^{2}+\frac{28}{125} z-\frac{239}{625}, \\
p_{3}(z)=\frac{8}{125} z^{3}+\frac{84}{625} z^{2}-\frac{114}{625} z-\frac{1,141}{3,125}, \\
p_{4}(z)=\frac{16}{625} z^{4}+\frac{224}{3,125} z^{3}-\frac{1,128}{15,625} z^{2}-\frac{21,448}{78,125} z+\frac{8,429}{78,125} .
\end{gathered}
$$
mation

The Theodorsenmethod with $N=1,024$ and $\omega=0.4$ gives, after $K=86$ iterations, the approxi-

\subsection{7}

to the capacity $c=2.5$ of the curve $\Gamma$. The corresponding approximations to the coefficients $c_{i}, j=0,1, \ldots, 20$ of the mapping function $\psi$ and the coefficients $\alpha_{k}^{\{20\}}, k=0,1, \ldots, 20$ of the Faber

polynomial $p_{20}(z)$ are listed in Tables 3.1 and 3.2 respectively. In each of the tables we also include the corresponding exact values which were obtained by rounding, if necessary, the rational MAPLE values to 10 significant figures.

3.3 Oval of Cassini Let $\Gamma$ be the oval of Cassini

$$
\begin{aligned}
& \Gamma:=\{\mathrm{z} \\
& \left.: \mathrm{z}=\rho(\theta) e^{i \theta}, 0 \leq \theta \leq 2 \pi\right\},
\end{aligned}
$$

where

$$
\rho(\theta):=\left\{\cos 2 \theta+\left[\cos ^{2} 2 \theta+a^{4}-1\right]^{1 / 2}\right\}^{1 / 2}, \quad 0 \leq \theta \leq \pi, \quad a \geq 1,
$$

and

$$
\rho(\theta)=\rho(2 \pi-\theta), \quad \pi<\theta \leq 2 \pi
$$

Then,

$$
\begin{gathered}
\psi(w)=a w \quad\left\{1+\frac{1}{a^{2} w^{2}}\right\}^{1 / 2}, \\
\phi(z)=\frac{z}{a}\left\{1-\frac{1}{z^{2}}\right\}^{1 / 2},
\end{gathered}
$$

and the exact value of $c:=\operatorname{cap} \Gamma$ is $c=a$.

For the case $a=2$, the Theodorsen method with $N=64$ and $\omega=1$ gives, after $K=18$ iterations, the capacity $\mathrm{c}=2$ correct to 14 significant figures. The corresponding approximations to the non-zero Laurent coefficients $c_{2 j-1}, j=1,2, \ldots, 10$ of the mapping function $\psi$ and the nonzero coefficients $\alpha_{2 k}^{\{20\}}, k=0,1, \ldots, 10$, of the Faber polynomial $p_{20}$ arelistedin Tables 3.3 and 3.4. The tables also include the corresponding exactvalues whichwere obtainedby rounding, if necessary, the rational MAPLE values to 12 significant figures.

Similarly, in the case $a=1.2$, the Theodorsen method with $N=64$ and $\omega=0.6$ gives, after $K=62$ iterations, the capacity c $=1.2$ correct to 13 significant figures. The corresponding approxima- tions to the non-zero coefficients $\mathrm{c}_{2 \mathrm{j}-1}, j=1,2, \ldots, 10$ and $\alpha_{2 k}^{\{20\}}, k=0,1, \ldots, 10$ are listed together with the exact values in Tables 3.5 and 3.6. 


\begin{tabular}{|c|l|c||c|c|c|}
\hline $\boldsymbol{J}$ & \multicolumn{1}{|c|}{${\text { Exact } \boldsymbol{c}_{\boldsymbol{j}}}$} & \multicolumn{1}{|c|}{ Approximate $\boldsymbol{c}_{\boldsymbol{j}}$} & $\boldsymbol{J}$ & Exart $_{\boldsymbol{j}}$ & ${\text { Approximate } \boldsymbol{c}_{\boldsymbol{j}}}$ \\
\hline \hline 0 & $-7.0(-1)$ & $-7.000000003(-1)$ & 11 & $2.496089277(-2)$ & $2.496089397(-2)$ \\
\hline 1 & $5.76(-1)$ & $5.760000008(-1)$ & 12 & $8.569622489(-3)$ & $8.569623452(-3)$ \\
\hline 2 & $1.6128(-1)$ & $1.612800008(-1)$ & 13 & $-1.532731879(-2)$ & $-1.532731942(-2)$ \\
\hline 3 & $-8.7552(-2)$ & $-8.755200040(-2)$ & 14 & $-1.458066666(-2)$ & $-1.458066803(-2)$ \\
\hline 4 & $-9.8832384(-2)$ & $-9.883238511(-2)$ & 15 & $5.053758185(-3)$ & $5.053757968(-3)$ \\
\hline 5 & $2.26639872(-3)$ & $2.266398481(-3)$ & 16 & $1.458799731(-2)$ & $1.458799856(-2)$ \\
\hline 6 & $5.747286344(-2)$ & $5.747286449(-2)$ & 17 & $3.277040132(-3)$ & $3.277041137(-3)$ \\
\hline 7 & $2.473365366(-2)$ & $2.473365457(-2)$ & 18 & $-1.059436651(-2)$ & $-1.059436713(-2)$ \\
\hline 8 & $-2.677287058(-2)$ & $-2.677287114(-2)$ & 19 & $-8.273365964(-3)$ & $-8.273367367(-3)$ \\
\hline 9 & $-3.005744396(-2)$ & $-3.005744525(-2)$ & 20 & $4.778735278(-3)$ & $4.778735005(-3)$ \\
\hline 10 & $4.934305707(-3)$ & $4.934305491(-3)$ & & & \\
\hline
\end{tabular}

Table 3.1 (Laurent coefs for 2 intersecting circles.)

\begin{tabular}{|c|c|c||c|c|c|}
\hline $\boldsymbol{K}$ & Exart $\alpha_{k}^{\{20\}}$ & Approximate $\alpha_{k}^{\{20\}}$ & $\boldsymbol{K}$ & Exart $\alpha_{k}^{\{20\}}$ & Approximate $\alpha_{k}^{\{20\}}$ \\
\hline \hline 0 & $1.608445417(-1)$ & $1.608445650(-1)$ & 11 & $-1.165648426(-3)$ & $-1.165648442(-3)$ \\
\hline 1 & $6.637133211(-2)$ & $6.637132432(-2)$ & 12 & $-1.339408792(-5)$ & $-1.339408918(-5)$ \\
\hline 2 & $-1.000767335(-1)$ & $-1.000767435(-1)$ & 13 & $2.183991056(-4)$ & $2.183991076(-4)$ \\
\hline 3 & $-4.230563949(-2)$ & $-4.230563472(-2)$ & 14 & $4.278154752(-5)$ & $4.278154797(-5)$ \\
\hline 4 & $4.955265047(-2)$ & $4.955264757(-2)$ & 15 & $-2.201457486(-5)$ & $-2.201457499(-5)$ \\
\hline 5 & $2.383175181(-2)$ & $2.383175556(-2)$ & 16 & $-9.410538603(-6)$ & $-9.410538664(-6)$ \\
\hline 6 & $-1.894848188(-2)$ & $-1.894848299(-2)$ & 17 & $8.774102790(-8)$ & $8.774102583(-8)$ \\
\hline 7 & $-1.124125419(-2)$ & $-1.124125409(-2)$ & 18 & $7.069859767(-7)$ & $7.069859795(-7)$ \\
\hline 8 & $5.213209722(-3)$ & $5.213209672(-3)$ & 19 & $1.539316279(-7)$ & $1.539316285(-7)$ \\
\hline 9 & $4.198521885(-3)$ & $4.198521940(-3)$ & 20 & $1.099511628(-8)$ & $1.099511632(-8)$ \\
\hline 10 & $-8.358293364(-4)$ & $-8.358293397(-4)$ & & & \\
\hline
\end{tabular}

Table 3.2 (Faber poly. for $2_{20}$ intersecting circles 
$-10-$

\begin{tabular}{|c|c|c|}
\hline$J$ & \multicolumn{1}{|c|}{ Exact $_{j}$} & Approximate $c_{j}$ \\
\hline \hline 1 & $2.5(-1)$ & $2.50000000000(-1)$ \\
\hline 3 & $-1.5625(-2)$ & $-1.56250000000(-2)$ \\
\hline 5 & $1.953125(-3)$ & $1.95312500004(-3)$ \\
\hline 7 & $-3.0517578125(-4)$ & $-3.05175781194(-4)$ \\
\hline 9 & $5.34057617188(-5)$ & $5.34057616558(-5)$ \\
\hline 11 & $-1.00135803223(-5)$ & $-1.00135803497(-5)$ \\
\hline 13 & $1.96695327759(-6)$ & $1.96695333867(-6)$ \\
\hline 15 & $-3.99537384510(-7)$ & $-3.99537389314(-7)$ \\
\hline 17 & $8.32369551063(-8)$ & $8.32369199513(-8)$ \\
\hline 19 & $-1.76878529601(-8)$ & $-1.76878371048(-8)$ \\
\hline
\end{tabular}

Table 3.3 (Laurent coefs for oval of Cassini $a=2.0)$

\begin{tabular}{|c|c|c|}
\hline$k$ & Exact $\alpha_{k}^{\{20\}}$ & Approximate $\alpha_{k}^{\{20\}}$ \\
\hline \hline 0 & $9.536743164(-7)$ & $9.53674118135(-7)$ \\
\hline 2 & $-9.536743164(-6)$ & $-9.53674309912(-6)$ \\
\hline 4 & $4.2915344238(-5)$ & $4.29153442649(-5)$ \\
\hline 6 & $-1.14440917968(-4)$ & $-1.14440917976(-4)$ \\
\hline 8 & $2.00271606444(-4)$ & $2.00271606443(-4)$ \\
\hline 10 & $-2.40325927733(-4)$ & $-2.40325927733(-4)$ \\
\hline 12 & $2.00271606444(-4)$ & $2.00271606445(-4)$ \\
\hline 14 & $-1.14440917968(-4)$ & $-1.14440917969(-4)$ \\
\hline 16 & $4.2915344238(-5)$ & $4.29153442383(-5)$ \\
\hline 18 & $-9.53674316406(-6)$ & $-9.53674316406(-6)$ \\
20 & $9.53674316406(-7)$ & $9.53674316406(-7)$ \\
\hline
\end{tabular}

Table 3.4 (Faber poly. $p_{20}$ for oval of Cassini $\mathrm{a}=2.0$ ) 


\begin{tabular}{|c|c|c|}
\hline$j$ & Exact $_{j}$ & Approximate $\mathbf{c}_{\mathrm{j}}$ \\
\hline \hline 1 & $4.16666666667(-1)$ & $4.16666666666(-1)$ \\
\hline 3 & $-7.23379629629(-2)$ & $-7.23379629632(-2)$ \\
\hline 5 & $2.51173482510(-2)$ & $2.51173482519(-2)$ \\
\hline 7 & $-1.09016268451(-2)$ & $-1.09016268458(-2)$ \\
\hline 9 & $5.29940193857(-3)$ & $5.29940193841(-3)$ \\
\hline 11 & $-2.76010517634(-3)$ & $-2.76010517509(-3)$ \\
\hline 13 & $1.50600976884(-3)$ & $1.50600976708(-3)$ \\
\hline 15 & $-8.49745095263(-4)$ & $-8.49745095234(-4)$ \\
\hline 17 & $4.91750633833(-4)$ & $4.91750633546(-4)$ \\
\hline 19 & $-2.90269471360(-4)$ & $-2.90269471928(-4)$
\end{tabular}

Table 3.5 (Laurent coefs for oval of Cassini a=1 .2 )

\begin{tabular}{|c|c|c|}
\hline$K$ & Exact $\alpha_{k}^{\{20\}}$ & Approximate $\alpha_{k}^{\{20\}}$ \\
\hline \hline 0 & $2.608405331(-2)$ & $2.60840533044(-2)$ \\
\hline 2 & $-2.608405331(-1)$ & $-2.60840533003(-1)$ \\
\hline 4 & 1.17378239895 & $1.17378239867(-1)$ \\
\hline 6 & -3.1300863972 & -3.13008639656 \\
\hline 8 & 5.4776511951 & 5.47765119398 \\
\hline 10 & -6.57318143412 & -6.57318143275 \\
\hline 12 & 5.47765119510 & 5.47765119395 \\
\hline 14 & -3.13008639720 & -3.13008639654 \\
\hline 16 & 1.17378239895 & 1.17378239870 \\
\hline 18 & $-2.608405331(-1)$ & $-2.60840533045(-1)$ \\
\hline 20 & $2.608405331(-2)$ & $2.60840533045(-2)$ \\
\hline
\end{tabular}

Table 3.6 (Faber poly. $\mathrm{p}_{20}$ for oval of Cassini $\mathrm{a}=1.2$ ) 
3.4 Circular sectors Let $\Omega_{1}$ be a circular sector of the form

$$
\{\zeta:|\zeta|<1, \quad|\arg \zeta|<\alpha\}, \quad 0<\alpha<\pi / 2
$$

For the application of the Theodorsen method we translate $\Omega_{1}$ along the real axis ( so that the vertex at $\zeta=0$ goes to the point $\mathrm{z}=0.5$ ) and express the boundary $\Gamma$ in polar form:

$$
\Gamma:=\left\{\mathrm{z}-. \mathrm{z}=\rho(\theta) \mathrm{e}^{\mathrm{i} \theta}, 0 \leq \theta \leq 2 \pi\right\},
$$

where

$$
\rho(\theta):= \begin{cases}0.5\left\{-\cos \theta+\left[\cos ^{2} \theta+3\right]^{1 / 2}\right\}, & 0<\theta \leq \pi-\beta, \\ 0.5 \sin \alpha / \sin (\theta-\alpha), & \pi-\beta<\theta \leq \pi,\end{cases}
$$

with

$$
\rho(\theta)=\rho(2 \pi-\theta), \quad \pi<\theta \leq 2 \pi \pi
$$

And

$$
\beta:=\operatorname{arc} \tan \left\{\frac{2 \sin \alpha}{1-2 \cos \alpha}\right\}
$$

A formula for the conformal mapping $\vee /$ and a recurrence relation for the coefficients of its LaurentexpansionhavebeenderivedrecentlybyColeman and Smith in [2]. In particular, it is shown in [2] that the capacity of $\mathrm{F}$ is given by

$$
c=\frac{\hat{\alpha}^{2}}{(2 \hat{\alpha}-1)^{2-1 / \hat{\alpha}}}, \quad \text { Where } \quad \hat{\alpha}:=\pi / \alpha .
$$

Our numerical results are for the three cases $\alpha=\pi / 2, \pi / 4$ and $\pi / 12$. The values of the degree $N$ of the interpolating polynomial, the relaxation parameter $\omega$ and the number of iterations $K$ used, in each case, are given below together with the value of $c:=$ cap $\Gamma$ and the computed approximation $\tilde{c}$ - (Here, for eacha, the relaxation parameter co was determined as indicated by Gutknecht [10, p.412], by estimating the value of e in (2.19) and taking $\omega=1 /\left(1+\varepsilon^{2}\right)$.) The corresponding approximations to the Laurent coefficients $c_{j,} j=0,1, \ldots, 10$, of the mapping function $\psi$, and the coefficients $\alpha_{i}^{\{10\}}, j=0,1, \ldots, 10$, of the Faber polynomial $\mathrm{p}, 0$, are listed in Tables $3.7-3.12$.

$$
\begin{gathered}
\text { (i) } \alpha=\pi / 2 \\
N=2,048, \quad \omega=0.2, \quad K=114, \\
\mathrm{c}=0.769800359, \quad \tilde{c}=0.769800296 .
\end{gathered}
$$

(ii) $\alpha=\pi / 4$

$$
\begin{aligned}
& N=2,048, \quad \omega=0.23, \\
& K=100, c=0.531127041, \quad \tilde{\mathrm{c}}= \\
& 0.531127014 .
\end{aligned}
$$




$$
\begin{gathered}
\text { (iv) } \alpha=\pi / 12 \\
N=2,048, \quad \omega=0.05895, \quad K=288, \\
c=0.353495704, \quad \tilde{c}=0.353495718 .
\end{gathered}
$$

For comparison purposes, in each of the Tables 3.7, 3.9, and 3.11 we include the exact values of the Laurent coefficients $c_{j}, j=0,1, \ldots, 10$, rounded to eight decimal places. These were obtained by means of MAPLE from the formula for $\psi$ given in [2, p.232]. Similarly, in each of the Tables 3.8,

3.10 , and 3.12 we give the exact values of $\alpha_{i}^{\{10\}}, j=0,1 \ldots \ldots . .10$. These were obtained by rounding to

eight significant figures the appropriately normalized values given in the supplement of [2]. ( For the case $=\mathrm{n} / 2$ the exact $\alpha_{i}^{\{10\}}$ can also be determined, by means of MAPLE, from the exact formula for the mapping function $\phi$ which is given in [7, p.138].)

\subsection{Square Let $\Omega_{1}$ be the square}

$$
\Omega_{1}:=\{\mathrm{z}=, \mathrm{x}+\mathrm{iy}:|\mathrm{x}|<1,|\mathrm{y}|<1\} .
$$

Then,

$$
\begin{aligned}
\psi(w)= & c \int\left\{1+\frac{1}{w^{4}}\right\}^{1 / 2} d w \\
= & c\left\{w-\frac{1}{6} \frac{1}{w^{3}}+\frac{1}{56} \frac{1}{w^{7}}-\frac{1}{176} \frac{1}{w^{11}}+\frac{1}{384} \frac{1}{w^{15}}-\frac{7}{4,864} \frac{1}{w^{19}}\right. \\
& \left.+\frac{21}{23,552} \frac{1}{w^{23}}-\frac{33}{55,296} \frac{1}{w^{27}} \ldots .\right\},
\end{aligned}
$$

where to 11 decimal places the exact value of cap $\Gamma$ is

$$
\mathrm{c}=1.18034059902 \text {, }
$$

( see e.g. [1], [5, p.582] and [7, p.114]).

In this case the Theodorsen method with $N=2,048$ and $\omega=0.5$ gives, after $K=46$ iterations, the approximation

$$
1.18034071
$$

to the capacity $c=1.18034060$. The corresponding approximations to the non-zero coefficients $c_{j} ; j=3$ (4)23 of $\psi$ are listed in Table 3.13 and are compared with the exact values obtained from (3.4). In Table 3.14 we list the approximations to the non-zero coefficients $\alpha_{k}^{\{18\}}, k$ = 2 (4) 18 of the Faber polynomial $p$. These are compared with the corresponding exact values, which were obtained by means of MAPLE from the exact formula for $\phi$ given by Elliot in [7, $p$. $142]$. 


\begin{tabular}{|c|c|c||c|c|c|}
\hline$j$ & Exact $_{j}$ & Appromimate $c_{i}$ & $j$ & Exact $c_{j}$ & Approximate $c_{j}$ \\
\hline 0 & 0.38490018 & 0.38490024 & 6 & -0.00657788 & -0.00657785 \\
\hline 1 & -0.24056261 & -0.24056257 & 7 & -0.00071652 & -0.00071651 \\
\hline 2 & 0.12028131 & 0.12028122 & 8 & 0.00372941 & 0.00372938 \\
\hline 3 & -0.03909142 & -0.03909137 & 9 & -0.00234202 & -0.00234187 \\
\hline 4 & -0.00150352 & -0.00150350 & 10 & -0.00037555 & -0.00037367 \\
\hline 5 & 0.01165225 & 0.01165220 & & & \\
\hline
\end{tabular}

Table 3.7 ( Laurent coefs for sector; $\alpha=\pi / 2$ )

\begin{tabular}{|c|c|c|c|c|c|}
\hline$K$ & Extrat $\alpha_{k}^{\{10\}}$ & Approximate $\alpha_{k}^{\{10\}}$ & $K$ & Extrat $\alpha_{k}^{\{10\}}$ & Approximate $\alpha_{k}^{\{10\}}$ \\
\hline 0 & 1.0228004 & 1.0228001 & 6 & $2.1333436(+2)$ & $2.1333456(+2)$ \\
\hline 1 & -7.70174004 & -7.7017415 & 7 & $-1.8142044(+2)$ & $-1.8142061(+2)$ \\
\hline 2 & $2.9606695(+1)$ & $2.9606705(+1)$ & 8 & $1.1656898(+2)$ & $1.1656909(+2)$ \\
\hline 3 & $-7.4979728(+1)$ & $-7.4979764(+1)$ & 9 & $-5.2670449(+1)$ & $-5.2670501(+1)$ \\
\hline 4 & $1.3829487(+2)$ & $1.3829496(+2)$ & 10 & $1.368418(+1)$ & $1.3684195(+1)$ \\
\hline 5 & $-1.9472894(+2)$ & $-1.9472909(+2)$ & & & \\
\hline
\end{tabular}

Table 3.8 ( Faber poly. $p_{10}$ for sector; $\alpha=\pi / 2$ )

\begin{tabular}{|c|c|c||c|c|c|}
\hline$j$ & Exact $_{j}$ & Appromimate $c_{i}$ & $j$ & Exact $c_{j}$ & Approximate $c_{j}$ \\
\hline \hline 0 & 0.59751792 & 0.59751788 & 6 & -0.00717960 & -0.00717959 \\
\hline 1 & -0.09336218 & -0.09336208 & 7 & 0.00528546 & 0.00528542 \\
\hline 2 & -0.08169190 & -0.08169191 & 8 & 0.00058569 & 0.00021039 \\
\hline 3 & -0.05565261 & -0.05565258 & 9 & 0.00021106 & 0.00021039 \\
\hline 4 & -0.00453135 & -0.00453138 & 10 & -0.00251403 & -0.00252544 \\
\hline 5 & -0.00242124 & -0.00242120 & & & \\
\hline
\end{tabular}

Table3.9 (Laurent coefs sector ; $\alpha=\pi / 4$ ) 


\begin{tabular}{|c|c|c||c|c|c|}
\hline$k$ & Extra $\alpha_{k}^{\{10\}}$ & Approximate $\alpha_{k}^{\{10\}}$ & $K$ & Extra $\alpha_{k}^{\{10\}}$ & Approximate $\alpha_{k}^{\{10\}}$ \\
\hline \hline 0 & 1.5153427 & 1.5153351 & 6 & $1.7221208(+4)$ & $1.7221210(+4)$ \\
\hline 1 & $-4.9248911(+1)$ & $-4.9248819(+1)$ & 7 & $-1.5527986(+4)$ & $-1.5527990(+4)$ \\
\hline 2 & $4.7345174(+2)$ & $-4.7345125(+2)$ & 8 & $9.2711096(+3)$ & $9.2711128(+4)$ \\
\hline 3 & $-2.2966697(+3)$ & $-2.2966683(+3)$ & 9 & $-3.3447742(+3)$ & $-3.3447757(+3)$ \\
\hline 4 & $6.7506014(+3)$ & $6.7505993(+3)$ & 10 & $5.5977806(+3)$ & $5.5977834(+4)$ \\
\hline 5 & $-1.3057996(+4)$ & $-1.305799(+4)$ & & & \\
\hline
\end{tabular}

Table 3.10 (Faber poly. $\mathrm{P}_{10}$ for sector; $\boldsymbol{\alpha}=\boldsymbol{\pi} 4$ )

\begin{tabular}{|c|c|c||c|c|c|}
\hline$j$ & Extras $_{j}$ & Approximate $_{j}$ & $j$ & Extras $_{j}$ & Approximate $_{j}$ \\
\hline \hline 0 & 0.59406917 & 0.59406917 & 6 & 0.00343577 & 0.00343576 \\
\hline 1 & 0.15470551 & 0.15470552 & 7 & 0.00279611 & 0.00279596 \\
\hline 2 & -0.09115211 & 0.09115213 & 8 & -0.00000169 & -0.00000215 \\
\hline 3 & -0.01524769 & -0.01524770 & 9 & -0.00072477 & -0.00072816 \\
\hline 4 & -0.0495797 & -0.00495797 & 10 & -0.00133983 & -0.00133263 \\
\hline 5 & 0.00476505 & 0.00476506 & & & \\
\hline
\end{tabular}

Table 3.11 (Laurent coefs for sector ; $\boldsymbol{\alpha}=\boldsymbol{\pi} / 12$ )

\begin{tabular}{|c|c|c|c|c|c|}
\hline$k$ & Extra $\alpha_{k}^{\{10\}}$ & Approximate $\alpha_{k}^{\{10\}}$ & $\mathrm{k}$ & Extra $\alpha_{k}^{\{10\}}$ & Approximate $\alpha_{k}^{\{10\}}$ \\
\hline \hline 0 & 1.8443790 & 1.8443735 & 6 & 6.6921180 & $6.6921153(+5)$ \\
\hline 1 & $-1.8321334(+2)$ & $-1.832131(+2)$ & 7 & $-7.3671071(+5)$ & $-7.3671042(+5)$ \\
\hline 2 & $3.6684615(+3)$ & $3.6684589(+3)$ & 8 & $5.0329955(+5)$ & $5.0329935(+5)$ \\
\hline 3 & $-3.1087074(+4)$ & $-3.1087057(+4)$ & 9 & $-1.9498249(+5)$ & $-1.9498241(+5)$ \\
\hline 4 & $1.4173928(+5)$ & $1.4173921(+5)$ & 10 & $3.2821513(+4)$ & $3.282150(+4)$ \\
\hline 5 & $-3.877779(+5)$ & $-3.8777779(+5)$ & & & \\
\hline
\end{tabular}

Table 3.12 (Faber poly. $p_{10}$ for sector; $a=i t / 12$ ) 


\begin{tabular}{|r|r|r||l|r|r|}
\hline$j$ & \multicolumn{1}{|l|}{ Exact $_{j}$} & Approximate $c_{j}$ & $j$ & \multicolumn{1}{|c|}{${\text { Exact } c_{j}}$} & Approximate $c_{j}$ \\
\hline 3 & -0.19672343 & -0.19672361 & 15 & 0.00307380 & 0.00307387 \\
\hline 7 & 0.02107751 & 0.02107761 & 19 & -0.00169868 & -0.00169874 \\
\hline 11 & -0.00670648 & -0.00670656 & 23 & 0.00105244 & 0.00105250 \\
\hline
\end{tabular}

Table 3.13 (Laurent coefs for square )

\begin{tabular}{|c|c|c||c|c|c|}
\hline$K$ & Exact $\alpha_{k}^{\{18\}}$ & Approximate $\alpha_{k}^{\{18\}}$ & $k$ & Exact $\alpha_{k}^{\{18\}}$ & Approximate $\alpha_{k}^{\{18\}}$ \\
\hline & & & & & \\
2 & -0.00429094 & -0.00429132 & 14 & 0.29445465 & 0.29445448 \\
\hline 6 & 0.18676296 & 0.18676321 & 18 & 0.05056707 & 0.05056698 \\
\hline & & & & & \\
10 & 0.46267649 & 0.46267657 & & & \\
\hline
\end{tabular}

Table 3.14 ( Faber poly. $p_{18}$ for square )

\section{References}

[1] W.G. Bickley, Two-dimensional potential problems for the space outside a rectangle Proc. London Math. Soc., Ser. 2, 37 (1932) 82-105.

[2] J.P. Coleman and R.A. Smith, The Faber polynomials for circular sectors, Math. Comp. 49 (1987)231-241.

[3] J.H. Curtiss, Solutions of the Dirichlet problem in the plane by approximation with Faber polynomials, SIAM J. Numer. Anal. 3 (1966) 204-228.

[4] M. Eiermann, On semiiterative methods generated by Faber polynomials, Numer. Math. 56 (1989) 139-156.

[5] S.W. Ellacott, Computation of Faber series with application to numerical polynomial approximation in the complex plane, Math. Comp. 40 (1983) 575-587.

[6] S.W. Ellacott and E.B. Saff, On Clenshaws's method and a genaralization to Faber series, Numer. Math. 52(1988)499-509.

[7] G.H. Elliott, The construction of Chebyshev approximations in the complex plane (Ph.D Thesis, Faculty of Science, Mathematics, University of London 1978 ).

[8] D. Gaier, Konstruktive Methoden der konformen Abbildung ( Springer, Berlin 1964 ).

[9] D. Gaier, Lectures on Complex Approximation (Birkhauser, Boston-Basel-Stuttgart 1987). 
[10] M.H. Gutknecht, Solving Theodorsen's integral equation for conformal maps with the fast Fourier transform and various nonlinear iterative methods, Numer. Math. 36 (1981) 405 - 429.

[11] M.H. Gutknecht, Numerical conformal mapping methods based on function conjugation, in: $\quad$ L. N. Trefethen, Ed., Numerical Conformal Mapping (North-Holland, Amsterdam 1986) 31-77.

[12] P. Henrici, Applied and Computational Complex Analysis, Vol. III (Wiley, New

York1986). [13] H. Kober, Dictionary of conformal representations (Dover, New York $1957)$. 


\section{NOT TO BE REMOVED}

FROM THE LIBRARY

XB 23214473

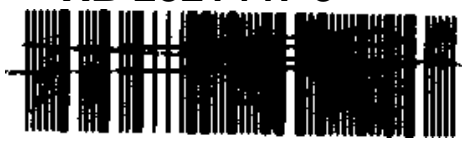

